\title{
An Experimental Study on the Application of Ultrasonic Technology for Demulsifying Crude Oil and Water Emulsions
}

Mahmood Amani*, Idris M, Abdul Ghani M, Dela Rosa N, Carvero A and Yrac R

Texas A\&M University at Qatar, Qatar

\begin{abstract}
An emulsion is the mixture of two immiscible fluids, where one fluid appears as droplets within another. In the oil and gas industry, produced crude oil generally comes with an appreciable amount of water within it in an emulsified form. Before produced crude oil can be prepared for purchase, the water associated with it must be removed. A process known as demulsification is required in order to separate an emulsion into its two phases. In the industry, a number of demulsification techniques are already present; these include thermal, mechanical, chemical, and electrical techniques.

Crude oil and gas produced from wells originally come with water, salts, and volatile gases such as oxygen, carbon dioxide, and sometimes hydrogen sulfide, etc. Hence, the petroleum mixture needs to be refined-water, salt, and non-hydrocarbon gases to be separated from the mixture, in order to meet certain oil and gas specifications (which state the maximum concentrations of such contaminants) and make it ready for purchase and transportation.

Sonication provides a cheap, simple, and harmless (as it involves mainly the propagation of sound waves) way of separating crude oils from water droplets via demulsification. In addition, if needed, it can be used for emulsification processes as well. Hence, a study of sonification as a way for crude refinement or chemical mixing has important implications for the oil and gas. This investigation proposes the use of ultrasonication as a new and cost-effective technique to aid in the demulsification of crude oil emulsion. The effectiveness of this technique was gauged through its comparison to the already present methods in the industry. Based on the investigation it was found that centrifuge served as the best demulsification method for it reduced the turbidity by $86 \%$. In addition, the reduced turbidity achieved with proposed ultrasonication method ranges from $20 \%-60 \%$.
\end{abstract}

Keywords: Ultrasonic; Crude oil; Emulsions

\section{Significance}

In the production of crude oil, usually a significant amount of water is also produced. Many times, this water and oil mixture is in the form of emulsions. Emulsion separation is very time consuming, requires additional surface facilities and can be very costly. Any new method that can increase the efficiency of this process and or provide a cheaper method would be very much helpful to the petroleum industry.

\section{Objective}

The objective of this research is to investigate the potential use of ultrasonication as a new and cost-effective technique to aid in the demulsification of crude oil emulsion. This research is looking at various existing techniques and will focus on the use of ultrasonic waves as a potential technique for emulsion separation.

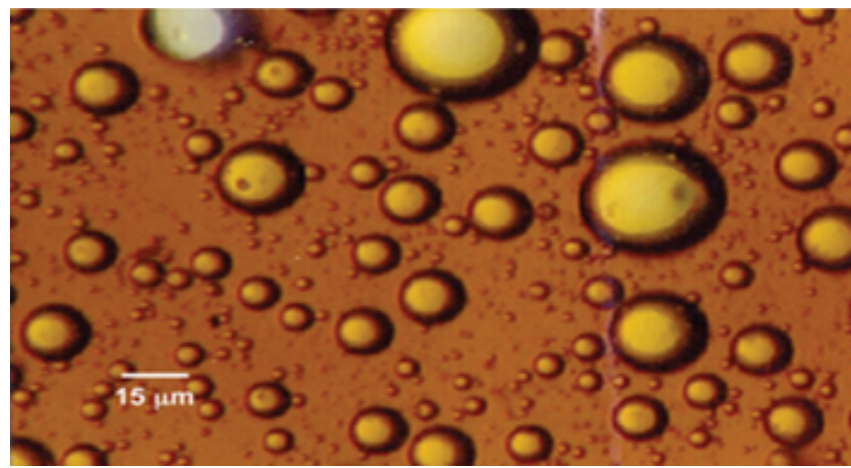

Figure 1: Photomicrograph of a W/O emulsion and a W/O/W emulsion.

\section{Introduction}

More than thirty percent of all crude oil produced in the world comes to the surface with an appreciable amount of water in an emulsified form [1]. A recurring issue in the oil industry is the separation of water from produced oil, its significance as an issue "is shown by an estimated 15-20 million dollars expended for chemicals each year to treat the world's oil production" [2]. An emulsion is defined as the dispersion of one liquid as droplets in another immiscible liquid. The water-inoil emulsion $(\mathrm{W} / \mathrm{O})$ that occurs because of crude oil production is the most common oil emulsion that is discussed in the industry and is the focus of this investigation; however, oil emulsions can come in many forms. The second most common form of oil emulsions is oil-inwater, sometimes referred to as reverse emulsions (Figure 1). Emulsions can also occur in more complex manners such water-in-oil-in-water, illustrated in Figure 2, where the droplets themselves house a third immiscible liquid.

In order to make the produced crude oil ready for purchase and transportation, the crude oil needs to be refined - water, other

"Corresponding author: Mahmood Amani, Texas A\&M University at Qatar, Qatar Tel: 974-5583-7368; E-mail: mahmood.amani@qatar.tamu.edu

Received May 25, 2017; Accepted June 15, 2017; Published June 21, 2017

Citation: Amani M, Idris M, Abdul Ghani M, Dela Rosa N, Carvero A, et al. (2017) An Experimental Study on the Application of Ultrasonic Technology for Demulsifying Crude Oil and Water Emulsions. J Pet Environ Biotechnol 7: 330. doi: 10.4172/2157-7463.1000330

Copyright: ( 2017 Amani M, et al. This is an open-access article distributed under the terms of the Creative Commons Attribution License, which permits unrestricted use, distribution, and reproduction in any medium, provided the original author and source are credited. 
nonpetroleum fluids, and solids to be separated from the mixture. Sonication is a cost-effective, simple, and safe (since it only involves sound wave propagation) means of separating water droplets from crude oil (demulsification). It may even be used for emulsification purposes as well. Therefore, sonification has important implications for the oil and gas industry.

We begin by discussing the characteristics and stabilizing components of emulsions. Second, we describe demulsification and the factors that affect such process. Third, we present the current demulsification methods used by the oil and gas industry. Fourth, we cover basic theory behind waves and sounds, and sonication. Finally, we examine sonication as means of demulsification.

\section{Emulsion}

Emulsions can be characterized by their kinetic stability, their stability over a period. In other words, emulsions are grouped based on their separation rates. Based on kinetic stability, emulsions can be divided into three groups: loose, medium and tight emulsions. Loose emulsions can be thought of as highly unstable with a low separation time while tight emulsions are very stable with relatively high separation times. Kinetic stability of an oil emulsion is a result of two parameters: droplet size and the interfacial film surrounding the droplets. Droplet size is quite intuitive as smaller droplet sizes are a clear indication of a better size fluid and therefore a more stable emulsion. Likewise, the interfacial films that surround water droplets protect water droplets from combining with each other and thus increasing the emulsion stability. More viscous interfacial films lead to more stable oil emulsions. The interfacial films around the water droplets play the largest role in creating a stable oil emulsion. Interfacial films with higher viscosity work the best in preventing the emulsion in separating. These films can be enhanced with the addition of an emulsifying agent. Emulsifying agents are generally naturally occurring and come mixed with the produced crude oil. They can be divided into two main categories: surfactants and fine solids. Surfactants work by attaching to the oil water interface, due to their unique affinity to both oil and water, where they form an interfacial film. Examples of surfactants are asphaltenes and resins. Fine solids work to stabilize emulsions by providing a mechanical barrier in between the droplets in addition to the interfacial film. These solids are most effective when they are smaller than the dispersed droplets. Examples of fine solids found in crude oil emulsions include clay, sand, and silt.

Given a mixture of two immiscible fluids, mechanical agitation, or disturbance, causes dispersion of one phase as tiny droplets (dispersants) throughout the other continuous liquid phase, and

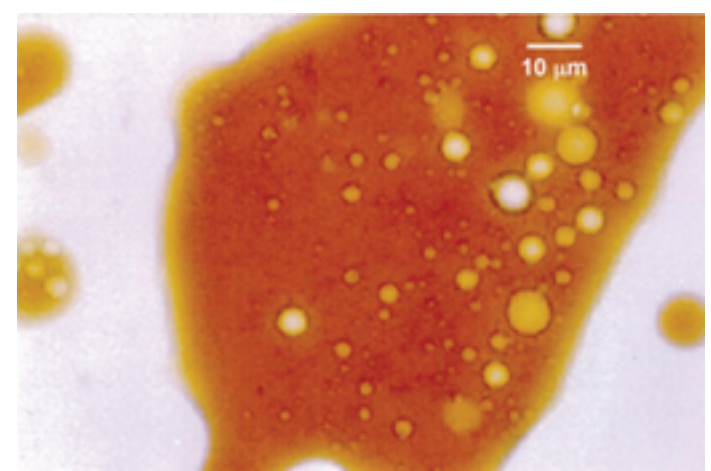

Figure 2: Photomicrograph of a W/O emulsion and a W/O/W emulsion.

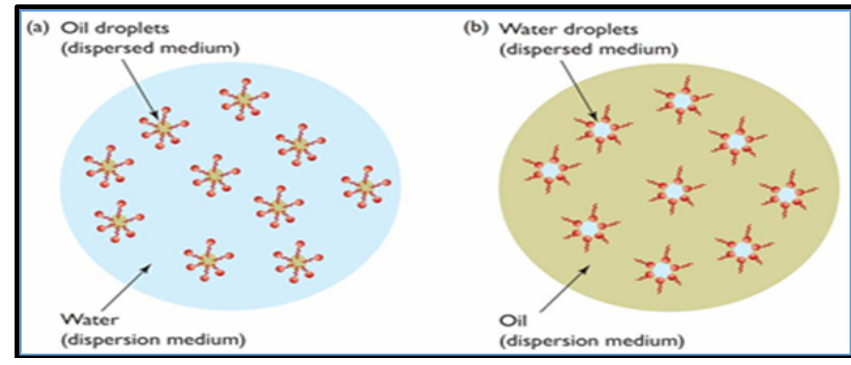

Figure 3: Types of emulsion.

subsequently the formation of the interfacial films of surfactants around the dispersed droplets for stabilizing an emulsion. Hence, sound wave propagation, which causes agitation via pressure fluctuations, can be used for emulsification purposes such as mixing viscosifier and oil into water-based drilling muds to obtain appropriate rheological properties (Figure 3).

\section{Demulsification of crude oil (water-in-oil emulsion)}

Demulsification is the separation of the emulsion into its separate components (water and oil). Demulsification as a treatment process is defined by the:

\section{Rate of separation}

\section{Remaining water/salt content in oil.}

\section{Remaining oil content in water}

Ideally, an optimum demulsification method would separate the crude oil while maximizing on these three parameters. Reduction of the rate of separation will aid in maximizing profits by increasing the efficiency of the treatment process. Moreover, the goal of any demulsification is to eliminate the water in oil emulsion that occurs as part of the desalting process (intentional mixing of crude and fresh water) as well as the emulsion with the water content that comes naturally with produced crude. The separation of the water droplets containing dissolved salt is ideal in avoiding production problems including "corrosion, scale accumulation, lowering of activity analysts and plugging or fouling in pipeline [3]. Moreover, demulsification is critical in achieving a marketable standard of crude oil. Finally, ideal demulsification would see that the separated water phase is composed of low oil content so that its disposal or reuse satisfies the environmental standards established by the industry.

Demulsification can be defined as breaking emulsion kinetic stability. As a result, demulsification can be said to occur in a twostep process consisting of flocculation and coalescence. Flocculation involves the gathering of water particles to near proximity of each other, while coalescence occurs when the individual films holding the water droplets break to allow the droplets to combine and culminate into bigger drops. Neither of these processes occurs faster than the other; however flocculation general must occur first before coalescence. As a result, in designing a demulsification program one must subject the emulsion to a combination of treatments that enhance both the flocculation and coalescence of the water droplets. Table 1 provides a list of the factors that affect both of these processes'. It is important to note that a high flocculation will aid in enhancing coalescence.

\section{Curent demulsification techniques}

Demulsification programs are very specific to the oil field and the crude oil that is produced. However, based on the factors presented in 


\begin{tabular}{|c|c|}
\hline $\begin{array}{c}\text { Factors that enhance flocculation } \\
\text { Amount of water in the emulsion }\end{array}$ & $\begin{array}{c}\text { Factors that enhance coalescence } \\
\text { High temperatures } \\
\text { Low oil viscosity }\end{array}$ \\
$\begin{array}{c}\text { Hensity difference between the two } \\
\text { fluids }\end{array}$ & Low oil viscosity \\
\hline Electrostatic field & High temperatures \\
\hline
\end{tabular}

Table 1: Factors that enhance the rate of flocculation and coalescence of water droplets [4]

Table 1, there are a number of common types of methods to treat an emulsion. Emulsion treatment methods can be grouped into four main categories; these are thermal, mechanical, electrical, and chemical.

Thermal: The application of thermal energy is probably the simplest method of demulsification used in the application of thermal energy is probably the simplest method of demulsification used in the industry. Thermal energy reduces the viscosity of the oil and increases the water settling rates." [4]. In addition, the increased temperatures "also result in the destabilization of the rigid films caused by reduced interfacial viscosity" and in some cases even rupturing of the films which in turn aids in the coalescence of the water particles [4]. The increased temperature also leads to an overall increase of kinetic energy of the particles leading to a higher coalescence frequency of the water droplets because of their increased mobility. In other words, heat accelerates the demulsification process in a variety of ways, however it is important to note that heat alone is not known to completely separate an emulsion on its own especially when dealing with stable emulsions. In fact, the application of thermal energy to an oil emulsion can lead to some undesirable effects, such as a reduction of the API gravity, and an "increased tendency for corrosion and scale deposition in treating vessels" [4]. In light of this, it's always important to weigh the costs of heating an oil emulsion against the adverse effects of the treatment in deciding whether thermal energy is an economic demulsification technique for the given emulsion case.

Electrical: An electrical demulsification method that is commonly used in the industry involves the application of an electric field perpendicular to the direction of flow. The associated charge of the water droplets along with the electric field can result in one of three main phenomena in occurring, all of which aid in destabilizing the emulsion. These phenomena include:

- The electric field aligns the water droplets based on polarity, thus positive ends of droplets are brought next to negative ends of other droplets. Through electrostatic attraction these droplets come closer and closer together eventually leading them to coalesce

- The polarity caused by an electric field can also make water particles attracted to an electrode. This can lead to large amount of water particles gathering and collecting in one-area allowing forming larger water droplets and eventually settling and separating from the oil.

- When an AC field is applied to an oil emulsion that effect is a weakening or even rupturing of the film surrounding the water droplets. This occurs because of the cyclic nature of an AC electrostatic grid. During the high voltage, phase of this cycle the water droplets are elongated along either end of the droplet, when the cycle returns back to the low voltage phase the droplets snap back to a spherical shape.

Regardless of which of the three phenomena occur when an electrostatic grid is applied to an oil emulsion the result is an acceleration of the demulsification process. An umbrella term for all three phenomena is known as Electrostatic Dehydration. This method is best used in combination with chemical or thermal techniques [5].
Chemical: The most common of the four techniques is the use of chemicals, known as demulsifiers, in treating an oil emulsion. Demulsifiers work by breaking the interfacial film protecting the trapped water droplets. In order to optimize the use of demulsifiers a number of factors are considered before the addition of a demulsifier into a crude oil emulsion including, proper selection of demulsifier accurate dosage, and adequate mixing time.

\section{Demulsifier selection}

In general, demulsifiers can be broken down to 3 critical components; solvents, surface-active ingredients, and flocculants. Over the years the selection of chemicals used as demulsifiers in the industry has vastly increased. With such a large number of options available it's very important that the right chemical combinations are selected in order to ensure the best results. Demulsifier selection begins first with characterization of the crude oil emulsion in question; breaking down the type of crude oil/brine and all the contaminants/ solids that make up the emulsion. Next, the production conditions of the crude oil emulsion should be considered including operating temperatures, production rates, treating vessel characteristics etc. Finally, it's important to consider any past record on the performance of the demulsifier with other crude oil emulsions. Demulsifier selection is always a unique experience and can vary considerably between different well case studies.

\section{Demulsifier dosage and mixing time}

Similar to demulsifier selection, demulsifier dosage is unique and depends entirely on the chosen demulsifier and the properties of the emulsion. Proper dosage is highly important in ensuring an optimized treatment of the emulsion. In fact, while too small of a demulsifier dosage will leave an emulsion as is, too much demulsifier can actually result in a more stable emulsion. Again, due to the large number of factors that can vary significantly between scenarios it's important to conduct accurate testing with the case in question in determining the optimum dosage. Equally as important is ensuring the chosen demulsifier is mixed adequately into the emulsion. Demulsifiers work by attaching to the oil and water interface and therefore the mixture has to be subjected to enough agitation in order to allow the demulsifier to reach the interfacial film. However, an unnecessary amount of mixing can reverse the effects of the demulsifier and stabilize the emulsion. Therefore, it's important to monitor the mixing of the demulsifier into the emulsion and decrease agitation significantly once the demulsifier begins to break the emulsion.

\section{Mechanical}

There are a variety of emulsions treating equipment that can be used to emulsify an oil emulsion. Some of the common ones used in the industry include: free knock out water drums; two/three phase separators, desalters, and settling tanks.

\section{Free knock out water drums}

Aside from taking out water from crude oil, free-water knockout drums can also separate associated gases. This equipment usually supplements main demulsification equipment.

\section{Three-phase separators}

As its name suggests, the three-phase separator separates the produced crude oil into three phases existing within the crude-oil, water, and gas. They can be set up vertically or laterally. The separator is appropriately assigned set retention time for sufficient separation at any 
required throughput rate. The separator can have components of heat section, wash water, filter section, stabilizing section, and electrostatic grids. It is no surprise then that large varieties of separators are in used today. What specific separator design would a demulsification task require is a complicated engineering task, which must consider many factors?

A flexible separator design, which allows for modification, is most preferable. Pressures and temperature conditions, water cuts, and oil and brine compositions change over the life of the field; hence, a well-designed separator is one that allows for adaptations to these fluctuating operation conditions.

Coalescer packs are a good way to increase separator efficiency. These packs increase the amount of fluid traversing through the separator. Water droplets coalesce as the emulsion rolls or wipes through the packing. Installation of spreaders, which increase droplet collision frequency, are also viable choice for improving separation efficiency.

\section{Desalters}

Separators usually are not enough to reduce the amount of water in the crude oil to marketable standards. In addition, some salt must be taken out as well. Hence, a desalter must be employed for removing the remaining water and salts. Desalters usually employ combinations of settling, chemical addition, and electrostatic treating. The time for settling depends on oil specification. With chemical addition, fresh water is also added for decreasing salt concentration (i.e. dilution) in the effluent water and crude. A one-stage desalter schematic is shown in Figure 4.

These are some of the most common techniques used in the industry to achieve demulsification. It is interesting to note that some techniques have overlapping components. For instance, desalters and three-phase separators may both employ a settling component and an electrostatic treating. Generally, a combination of these techniques is used in conjunction with each other to develop a complete demulsification program.

\section{Basic wave theory, sound, and sonication}

Harmonic motion: Harmonic motion is any repeating and oscillating motions. For instance, the back and forth motion of a mass attached to a fixed spring which is stretched, the swinging of a pendulum, and the motions of particles in a vibrating mass are all harmonic motions. An object in harmonic motion is an oscillator. A harmonic motion results from two things: A restoring force and inertia. A restoring force acts on the object in the direction towards equilibrium or decreasing potential energy. Inertia is the object's resistance to motion, or more specifically, the resistance to return to equilibrium. Hence these two-act opposite to each other in harmonic motion.

Period and frequency are the important parameters for describing the harmonic motions. Period $\mathrm{T}$ is the amount of time given in seconds (s). For one cycle of the motion to occur, and frequency $f$ is the number of cycles per unit time and is given in Hertz $(\mathrm{Hz}=1 / \mathrm{s})$. Also, they are related as:

\section{$\mathrm{T}=1 / \mathrm{f}$}

When the restoring force is directly proportional to the object's displacement, the motion is called simple harmonic motion (SHM). This can be stated as.

$$
F=-k x
$$

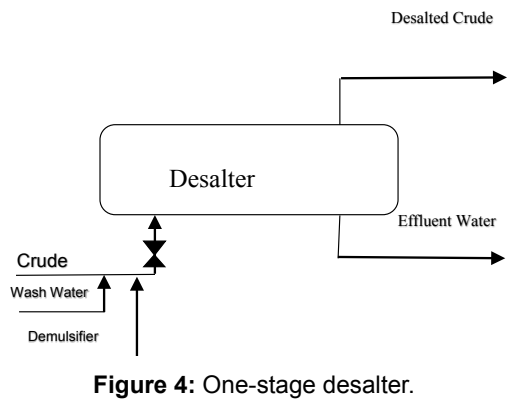

The minus sign is due to the restoring force acting opposite the displacement. The examples of harmonic motion given previously are all simple harmonic motions, although not necessarily for the vibrating mass case. Simple harmonic motions are described mathematically as sinusoidal functions:

$$
x(t)=A \cos (\omega t+\varphi)
$$

Where $\mathrm{x}, \mathrm{t}, \mathrm{A}, \omega$, and $\varphi$ are the displacement of the object, time, angular frequency, the constant called amplitude, and the constant called phase angle, respectively. The angular frequency is defined to be:

\section{$\Omega=2 \pi / T$}

Amplitude is the maximum displacement from equilibrium position of the object. For example, the amplitude for a swinging pendulum is the pendulum's maximum vertical displacement from its position when it is not in motion.

\section{Wave}

Waves are disturbances or oscillations carrying energy, and propagates through space (and are then electromagnetic waves), light for instance or mass (and are then called mechanical).

\section{Mechanical waves}

Mechanical waves are disturbances that propagate through matter, called the medium. As mechanical waves propagate continuously through the medium, the particles move roughly in a simple harmonic motion. Mechanical waves are generated for industrial or experimental purposes using simple harmonic oscillators.

The propagation speed of mechanical waves, or wave speed $\mathrm{v}$, increases with higher restoring forces $\mathrm{F}$, and decreases with higher inertia $\mu$. These relationships are more precisely given by:

$$
V=F / \mu
$$

\section{Transverse waves}

When the particles move perpendicular to the direction of wave propagation, the wave is transverse. Consider a string is fixed at one end to a wall and tied to a continuously moving oscillator in the other. Then as the wave generated by the oscillator propagates through the string, each of the string's particle move up and down, perpendicular to the wave direction. The collective motion of the particles results in a sinusoidal waving string. This is appropriately called a sinusoidal motion.

\section{Longitudinal waves}

When the particles move parallel to the direction of wave propagation, the wave is longitudinal. When a longitudinal wave propagates through a medium, particles move in or opposite the wave direction, and causing alternating regions of unidirectional rarefaction, low pressure, and compression, high pressure. 


\section{Sound}

Sounds are longitudinal mechanical waves. These are micro fluctuations in pressure that travel through a medium, commonly air. Our sensitive ears can detect these pressure fluctuations, which we then perceive as sound. The human ears can detect sound with frequency ranging from $20 \mathrm{~Hz}$ to $20,000 \mathrm{~Hz}$, called the audible range. A sound with frequency higher than $20,000 \mathrm{~Hz}$ are called ultrasonic.

\section{Sonication}

Sonication is simply the propagation of sound waves into a substance for agitation purposes. Ultrasonication uses sound waves of frequency above $20 \mathrm{KHz}$ (ultrasonic) and is commonly used. A sonication equipment may comprise of an electricity generator that generates AC electricity, a transducer that converts the AC electricity into mechanical vibrations, a transformer that amplifies this vibration, and a tool tip which makes the amplified vibration energy available for application (hence, tool tip is the oscillator to be applied) [1].

\section{Demulsification using ultrasonification}

Demulsification can be achieved using ultrasonification. Currently, there is not one unanimously agreed mechanism for which ultrasonification causes demulsification, although reasonable explanations have been proposed. The following is such an explanation [1]:

1. Ultrasound waves of high intensity produce cavitation or regions of free fluid spaces in the liquid.

2. Cavitation leads to rapid fluctuation of pressure.

3. Relatively large masses of vacuous, small bubbles form.

4. Bubbles grow until it reaches critical size.

5. Bubbles implode and generate intense shockwaves leading to high temperature and microstreaming of the liquid.

6. High shear gradient is produced throughout the mixture which weakens the interfacial films.

\section{Emulsion is destabilized.}

Demulsifiers are commonly employed for demulsification purposes, and ultrasonification can be used to complement them. Demulsification using demulsifiers is made more efficient through ultrasonification since the agitation due to ultrasonic waves aids in mixing the demulsifiers through the emulsion mixture better. This way, the demulsifiers may reach more of the interfacial oil-water film, drain the film of surfactants, and decrease the film's strength. This induces the coagulation of dispersed water blobs, and consequently the destabilizing of emulsion.

\section{Methodology}

As this project attempts to compare the efficiency of ultrasonication to other methods of oil demulsification, each of those techniques were performed separately. Before any experimentation was done

\begin{tabular}{|c|c|c|}
\hline \% Solution, oil in Water & Oil to Solvesso ratio & Highest turbidity, NTU \\
\hline 0.1 & $0.05: 0.05$ & 975 \\
\hline 0.08 & $0.04: 0.04$ & 713 \\
\hline 0.06 & $0.03: 0.03$ & 541 \\
\hline 0.04 & $0.02: 0.02$ & 252 \\
\hline 0.02 & $0.01: 0.01$ & 152 \\
\hline
\end{tabular}

Table 2: The base-turbidity of each emulsion.

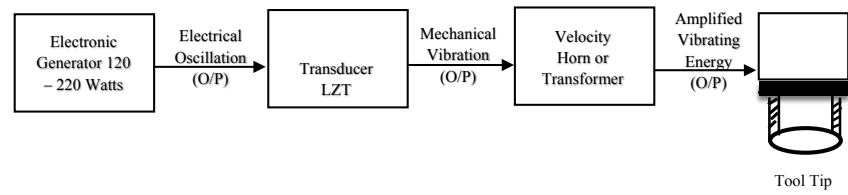

Figure 5: Diagram of sonication equipment setup.

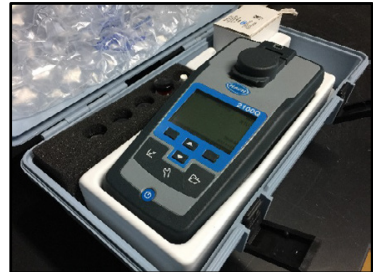

Figure 6: Hach 2100Q turbidimete

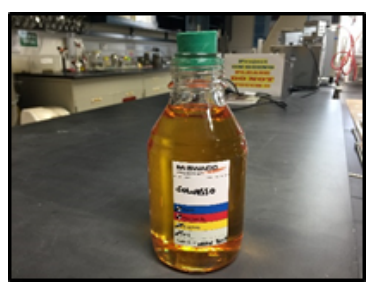

Figure 7: Solvesso 150.

to determine the demulsificating effect of any mechanism, turbidity for each oil-water concentration was obtained. This initial turbidity was then compared to the turbidity of the solution after any specific test was performed. From basic analysis, the test that results in the most turbidity decrease can be said to be the most effective test for demulsifying an emulsion.

\section{Base-turbidity determination}

To determine the base-turbidity, different concentrations of emulsions were created. To create each emulsion, studied concentrations of oil were added to a relevant amount of water. To form a stable emulsion, an emulsifier (Solvesso 150) was added alongside the oil after which rigorous mixing was done for 70 seconds with an electric solution mixer. This procedure was carried on for every emulsion created in this project. After mixing, the resulting solution was distributed into 5 beakers right after mixing and was allowed to set for $7 \mathrm{~min}$ before a turbidity reading was taken.

Many trials of this experiment were repeated and the concluded base turbidity for each mixture of different oil concentration is shown in Table 2. Turbidity reading was done on a turbidimeter from Hach Instruments Company (Figures 5-7).

\section{Emulsion breaker}

The concentration of the demulsifying agent which serves as a catalytic agent through time and external conditions such as temperature and pressure can satisfy and bridge to the comparison of all subjected samples.

As with the base turbidity test, emulsions were created by mixing different concentrations of oil and Solvesso 150 with water. In this test the following procedure was followed:

1. For a specific concentration of oil, an emulsion of around 1000 


\begin{tabular}{|c|c|c|c|c|c|c|c|c|c|c|}
\hline \multicolumn{2}{|c|}{ Concentration } & \multicolumn{9}{|c|}{ Turbidity, NTU } \\
\hline \multirow{2}{*}{$\begin{array}{c}\text { Oil in } \\
\text { water } \\
\%\end{array}$} & \multirow{2}{*}{$\begin{array}{c}\text { Emulsion } \\
\text { breaker } \\
\text { ppm }\end{array}$} & \multicolumn{3}{|c|}{ Trial 1} & \multicolumn{3}{|c|}{ Trial 2} & \multicolumn{3}{|c|}{ Trial 3} \\
\hline & & Initial & Final & \%Reduction & Initial & Final & \%Reduction & Initial & Final & \%Reduction \\
\hline \multirow{5}{*}{0.08} & 80 & 905 & 747 & 17 & 865 & 629 & 27 & 717 & 675 & 6 \\
\hline & 60 & 924 & 691 & 25 & 890 & 659 & 26 & 890 & 762 & 14 \\
\hline & 40 & 950 & 756 & 23 & 908 & 618 & 32 & 686 & 592 & 14 \\
\hline & 20 & 951 & 742 & 22 & 902 & 695 & 23 & 644 & 564 & 12 \\
\hline & 15 & 963 & 795 & 17 & 928 & 666 & 28 & 987 & 808 & 18 \\
\hline \multirow{5}{*}{0.06} & 80 & 618 & 614 & 1 & 492 & 477 & 3 & 656 & 642 & 2 \\
\hline & 60 & 571 & 578 & -1 & 489 & 462 & 6 & 629 & 696 & -11 \\
\hline & 40 & 579 & 551 & 5 & 337 & 327 & 3 & 599 & 592 & 1 \\
\hline & 20 & 555 & 525 & 5 & 336 & 300 & 11 & 611 & 599 & 2 \\
\hline & 15 & 527 & 493 & 6 & 337 & 293 & 13 & 594 & 546 & 8 \\
\hline \multirow{5}{*}{0.04} & 80 & 327 & 304 & 7 & 275 & 286 & -4 & 320 & 329 & -3 \\
\hline & 60 & 312 & 299 & 4 & 274 & 276 & -1 & 273 & 282 & -3 \\
\hline & 40 & 309 & 285 & 8 & 262 & 261 & 0 & 270 & 269 & 0 \\
\hline & 20 & 292 & 274 & 6 & 263 & 253 & 4 & 260 & 260 & 0 \\
\hline & 15 & 286 & 261 & 9 & 271 & 264 & 3 & 263 & 254 & 3 \\
\hline \multirow{5}{*}{0.02} & 100 & 118 & 138 & -17 & 117 & 126 & -8 & 202 & 214 & -6 \\
\hline & 80 & 123 & 123 & 0 & 172 & 179 & -4 & 190 & 197 & -4 \\
\hline & 60 & 125 & 115 & 8 & 172 & 166 & 3 & 192 & 187 & 3 \\
\hline & 40 & 125 & 106 & 15 & 167 & 156 & 7 & 192 & 182 & 5 \\
\hline & 20 & 118 & 99 & 16 & 168 & 160 & 5 & 178 & 174 & 2 \\
\hline
\end{tabular}

Table 3: The effect of different concentrations of emulsion breaker on different concentrations of emulsion.

\begin{tabular}{|c|c|c|c|c|}
\hline \multicolumn{2}{|c|}{ Concentration } & \multirow{2}{*}{\multicolumn{3}{|c|}{$\begin{array}{c}\text { Turbidity, NTU } \\
\text { Trial } 1\end{array}$}} \\
\hline Oil in water & Emulsion breaker & & & \\
\hline$\%$ & ppm & Initial & Final & \%Reduction \\
\hline \multirow{5}{*}{0.08} & 80 & 632 & 604 & 4.4 \\
\hline & 60 & 674 & 615 & 8.8 \\
\hline & 40 & 571 & 617 & -8.1 \\
\hline & 20 & 701 & 621 & 11.4 \\
\hline & 15 & 670 & 657 & 1.9 \\
\hline \multirow{5}{*}{0.06} & 80 & 420 & 376 & 10.5 \\
\hline & 60 & 463 & 396 & 14.5 \\
\hline & 40 & 422 & 389 & 7.8 \\
\hline & 20 & 411 & 399 & 2.9 \\
\hline & 15 & 445 & 428 & 3.8 \\
\hline \multirow{5}{*}{0.04} & 80 & 520 & 491 & 5.6 \\
\hline & 60 & 480 & 446 & 7.1 \\
\hline & 40 & 500 & 383 & 23.4 \\
\hline & 20 & 508 & 409 & 19.5 \\
\hline & 15 & 508 & 412 & 18.9 \\
\hline \multirow{5}{*}{0.02} & 80 & 92.2 & 91.2 & 1.1 \\
\hline & 60 & 85.5 & 92.1 & -7.3 \\
\hline & 40 & 88.7 & 90.6 & -2.1 \\
\hline & 20 & 92.4 & 88.7 & 4 \\
\hline & 15 & 98.8 & 94.3 & 4.6 \\
\hline
\end{tabular}

Table 4: The Effect of different concentrations of emulsion breaker on different concentrations of emulsion (continuation).

$\mathrm{mL}$ was created then immediately distributed in 5 beakers after mixing and left to set for $7 \mathrm{~min}$.

2. After setting for $7 \mathrm{~min}$, an initial turbidity reading was recorded and a noted amount of emulsion breaker was added.

3. The mixture of emulsion and emulsion breaker was mixed through a magnetic stirrer on $60 \mathrm{rpms}$ for $2 \mathrm{~min}, 40 \mathrm{rpms}$ for $2 \mathrm{~min}$ then $20 \mathrm{rpms}$ for the last $2 \mathrm{~min}$. A final turbidity was then recorded after mixing. This process was repeated for 3 trials. The results of all those trials are presented in Table 3.

\section{Water bath}

Since the density and viscosity depends on the temperature of the samples, this test aims to demulsify the samples by the function of time, temperature, viscosity and density.

To perform this section of the project, two things need to be optimized: the time and the temperature of exposure. To do that, the regular emulsion previously discussed was created and separated in different beakers. After taking and initial turbidity reading, these beakers were placed in a water bath at a certain temperature and each of them was left inside for a certain amount of time. After removal of the beakers from the water bath, the final turbidity reading was measured. This was repeated for different temperatures. The results of this are presented in Table 4.

This technique has been done in an SDM water bath device with a maximum heating temperature of $70^{\circ} \mathrm{C}$. This device is shown below on Figure 8.

\section{Centrifuge}

To test the demulsification efficiency of the centrifugal technique, the test needed to be optimized. For a fixed speed of $5000 \mathrm{rpm}$ and a

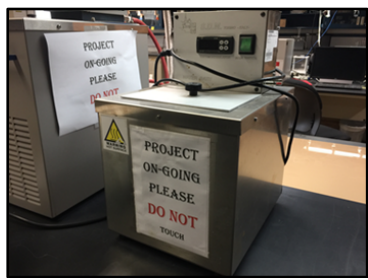

Figure 8: Water bath. 


\begin{tabular}{|c|c|c|}
\hline Oil in water, \% & EB concentration & \% Reduction \\
\hline 0.08 & 20 & 11 \\
\hline 0.06 & 60 & 15 \\
\hline 0.04 & 40 & 23 \\
\hline 0.02 & 15 & 5 \\
\hline
\end{tabular}

Table 5: The optimum emulsion breaker concentration for each oil in water concentration.

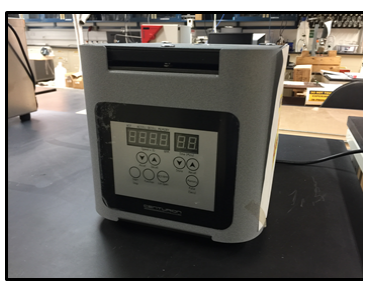

Figure 9: Centurion centrifuge.

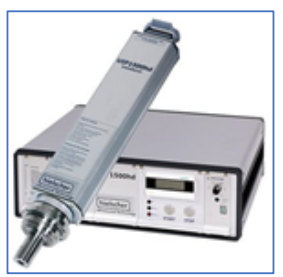

Figure 10: Ultrasonic device.

concentration of oil of 0.08 , the test was run for different durations. After finding the optimum duration for the test, emulsions of different concentrations were created then placed in centrifugal tubes and run for the optimum time at different rotation speeds. The reduction of turbidity of each trial was recorded and the results are presented in Table 5. This technique has been conducted on a Centurion Centrifuge with a rotation speed of $1000 \mathrm{rpm}$ to $5000 \mathrm{rpm}$ (Figure 9).

\section{Ultrasonication}

This test proposed several stages of time duration, temperature, amplitude and power to systematically determine the optimum level. This will provide a full analysis of the direct effect of ultrasonic waves on the emulsions and will determine the sonication durations and intensities that result in the most efficient separation.

This technique, an ultrasonic device from Hielscher Ultrasonics Model UIP1500hd has been used. This ultrasonic device has a maximum power of 1500 Watts and at $20 \mathrm{kHz}$. This device is also able to deliver up to 170 microns of Amplitude. Please refer to Figure 10.

\section{Results and Discussion}

For each of the four demulsification techniques performed the turbidity before and after were recorded for different operating conditions. The percentage reduction of turbidity for each test was computed to find the method resulting in the greatest reduction. This section Presents those collected results and the direct computations that stem from them.

\section{Base-turbidity determination}

The value of turbidity for each solution was found by taking the highest turbidity reading for each specific concentration during any of the trials performed. This follows the observation that as the oil, emulsifier, and water are mixed, splashes of water carry with them droplets of the oil that cause the emulsion to be of less concentration than the calculated value resulting in a value of turbidity less than the supposed value. The highest turbidity reading at any trial is then considered the reading for which the least splashing was done thus the most accurate. The value of this highest reading considered as the base turbidity for each concentration is presented in the following table.

These values of base turbidity were, however, disregarded as the experiment progressed. As it was observed, the turbidity of any solution changes with each trial so assigning a concentration a specific value of turbidity for the reduction to be compared with is not viable. Instead, a $\%$ decrease notation was followed in which the turbidity of each mixture is measured before any demulsification technique was applied to it. After the demulsification the turbidity was measured again and the $\%$ reduction in turbidity was noted. Some of the samples are shown on the photos below.

\section{Emulsion breaker}

The method involves using a specific amount of emulsion breaker or demulsifier to the predetermined oil in water emulsion concentrations. The emulsion breaker (EB-8956) used in this project is coming from a world leader emulsion breaker supplier in the industry MI Swaco. The first step taken was with determination of the optimum amount of emulsion breaker to be used for each concentration of oil in water emulsion. Please refer to Table 3.

Oil in water concentration was tested for 5 different concentrations of emulsion breaker ranging from 15 to $80 \mathrm{ppm}$ to find the optimum concentration for each concentration. This was first done over 3 trials which reported varying results that can be seen in Table 3 . The experiment was repeated one last time for a fourth trial to confirm the variations in the data. This last trial is represented in Table 4.

After finding the optimum concentration of emulsion breaker for oil in water concentration, Table 5 was created to summarize the results. In the table, the optimum emulsion breaker concentration alongside the \% reduction caused by it is presented. Kindly refer to the graphical representation Plot 1 of Table 5 below.

From these plots, we can deduce that as the concentration of the oil
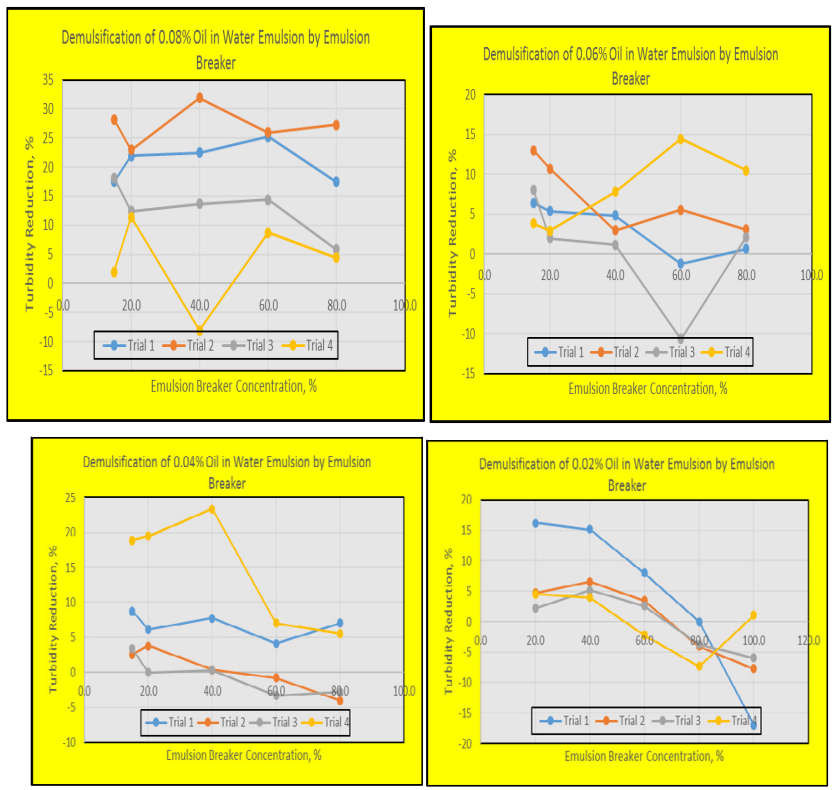

Plot 1: Demulsification of oil in water emulsion by emulsion breaker. 
Citation: Amani M, Idris M, Abdul Ghani M, Dela Rosa N, Carvero A, et al. (2017) An Experimental Study on the Application of Ultrasonic Technology for Demulsifying Crude Oil and Water Emulsions. J Pet Environ Biotechnol 7: 330. doi: 10.4172/2157-7463.1000330

Page 8 of 12

\begin{tabular}{|c|c|c|c|c|c|c|c|c|c|c|}
\hline \multirow{3}{*}{$\begin{array}{c}\text { Oil in water } \\
\text { concentration } \\
\%\end{array}$} & \multirow{3}{*}{$\begin{array}{c}\begin{array}{c}\text { Water bath } \\
\text { temperature }\end{array} \\
\text { Degree C }\end{array}$} & \multicolumn{9}{|c|}{ Turbidity, NTU } \\
\hline & & \multicolumn{3}{|c|}{ Trial 1} & \multicolumn{3}{|c|}{ Trial 2} & \multicolumn{3}{|c|}{ Trial 3} \\
\hline & & Initial & Final & \%Reduction & Initial & Final & \%Reduction & Initial & Final & \%Reduction \\
\hline \multirow{3}{*}{0.08} & 70 & 750 & 556 & 26 & 897 & 592 & 34 & 956 & 630 & 34 \\
\hline & 60 & 703 & 475 & 32 & 703 & 490 & 30 & 703 & 445 & 37 \\
\hline & 50 & 991 & 987 & 0 & 991 & 990 & 0 & 991 & 997 & -1 \\
\hline \multirow{3}{*}{0.06} & 70 & 739 & 514 & 30 & 739 & 512 & 31 & 739 & 504 & 32 \\
\hline & 60 & 483 & 323 & 33 & 483 & 313 & 35 & 483 & 341 & 29 \\
\hline & 50 & 799 & 793 & 1 & 799 & 804 & -1 & 799 & 789 & 1 \\
\hline \multirow{3}{*}{0.04} & 70 & 339 & 188 & 45 & 339 & 197 & 42 & 339 & 203 & 40 \\
\hline & 60 & 332 & 226 & 32 & 332 & 227 & 32 & 332 & 238 & 28 \\
\hline & 50 & 379 & 302 & 20 & 379 & 295 & 22 & 379 & 283 & 25 \\
\hline \multirow{3}{*}{0.02} & 70 & 140 & 91 & 35 & 140 & 90 & 36 & 140 & 93 & 34 \\
\hline & 60 & 151 & 110 & 27 & 151 & 109 & 28 & 151 & 116 & 23 \\
\hline & 50 & 95.6 & 74.1 & 22 & 95.6 & 69.6 & 27 & 95.6 & 70.8 & 26 \\
\hline
\end{tabular}

Note: Shaded cells highlight results with the highest turbidity reduction.

Table 6: The temperature optimization of the water bath.

\begin{tabular}{|c|c|c|c|c|c|c|c|c|c|c|c|c|}
\hline \multirow{3}{*}{ Time, mins } & \multicolumn{12}{|c|}{ Turbidity, NTU } \\
\hline & \multicolumn{3}{|c|}{ Trial 1} & \multicolumn{3}{|c|}{ Trial 2} & \multicolumn{3}{|c|}{ Trial 3} & \multicolumn{3}{|c|}{ Trial 4} \\
\hline & Initial & Final & \%Reduction & Initial & Final & \%Reduction & Initial & Final & \%Reduction & Initial & Final & \%Reduction \\
\hline 10 & 750 & 598 & 20 & 956 & 709 & 26 & 897 & 655 & 27 & & & \\
\hline 15 & 750 & 576 & 23 & 956 & 702 & 27 & 897 & 628 & 30 & & & \\
\hline 20 & 750 & 546 & 27 & 956 & 689 & 28 & 897 & 624 & 30 & & & \\
\hline 25 & 750 & 536 & 29 & 956 & 614 & 36 & 897 & 616 & 31 & & & \\
\hline 30 & 750 & 556 & 26 & 956 & 630 & 34 & 897 & 592 & 34 & & & \\
\hline 35 & 750 & 510 & 32 & 956 & 605 & 37 & 897 & 575 & 36 & & & \\
\hline 40 & 750 & 489 & 35 & 956 & 610 & 36 & 897 & 564 & 37 & & & \\
\hline 45 & & & & 956 & 597 & 38 & 897 & 545 & 39 & & & \\
\hline 50 & & & & & & & & & & 817 & 498 & 39 \\
\hline 55 & & & & & & & & & & 817 & 461 & 44 \\
\hline 60 & & & & & & & & & & 817 & 461 & 44 \\
\hline 65 & & & & & & & & & & 817 & 474 & 42 \\
\hline 70 & & & & & & & & & & 817 & 440 & 46 \\
\hline
\end{tabular}

Note: Empty cells do not have values due to sample limitations; shaded cells highlight highest turbidity reduction.

Table 7: The reduction in turbidity for $0.08 \%$ oil-in-water emulsion with time at $70^{\circ} \mathrm{C}$

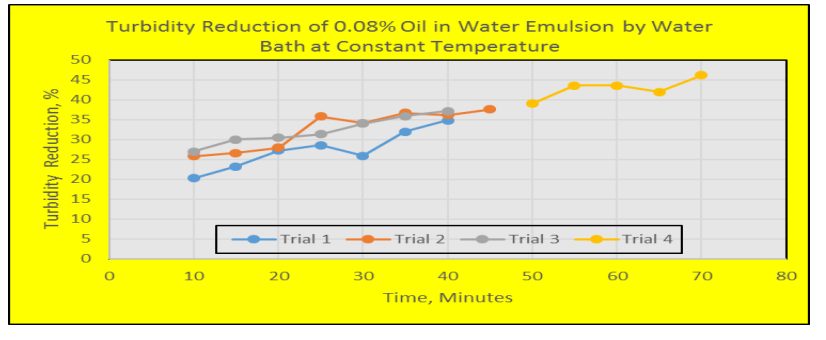

Plot 2: Turbidity reduction at constant temperature on a water bath.

in water emulsion sample reduces and as the concentration of emulsion breaker increases, sample turbidity increases.

\section{Water bath}

To test the reduction of turbidity used a water bath, two parameters needed to optimize: temperature and time. Firstly, four different concentrations of oil in water were mixed and placed in the water bath at different temperatures at $10^{\circ} \mathrm{C}$ increments from $40^{\circ} \mathrm{C}$ to $70^{\circ} \mathrm{C}$. The turbidity readings were taken before and after the treatment allowing for the calculation of the \% reduction in the turbidity. No reductions were found for $40^{\circ} \mathrm{C}$ water temperature, and the reductions at other temperatures are presented in Table 6.

As seen in the above table, the most reduction happened at a

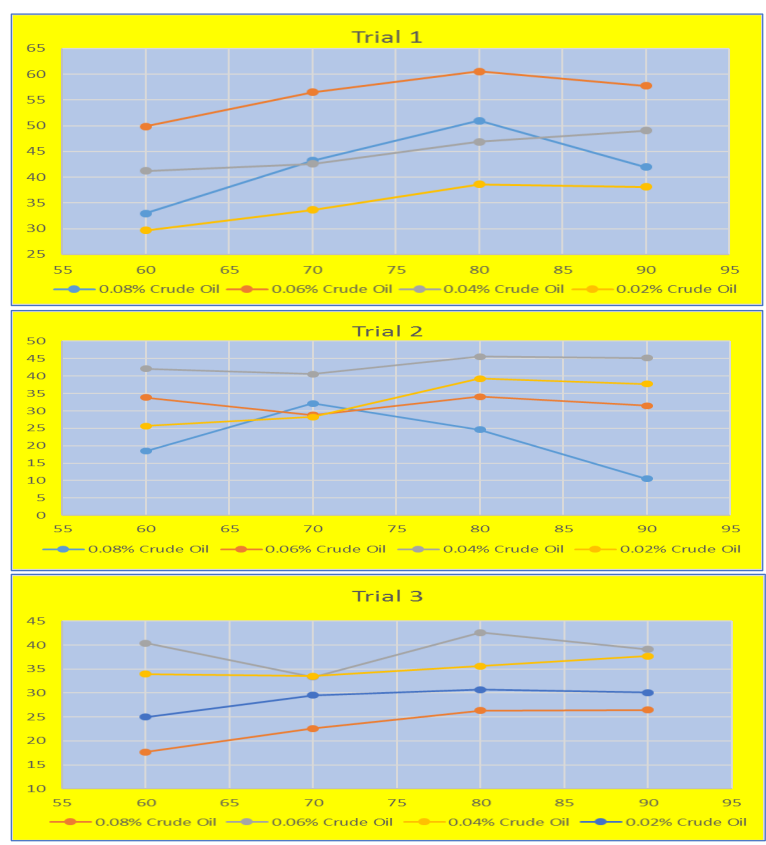

Plot 3: Percent turbidity reduction by ultrasonication. 
Citation: Amani M, Idris M, Abdul Ghani M, Dela Rosa N, Carvero A, et al. (2017) An Experimental Study on the Application of Ultrasonic Technology for Demulsifying Crude Oil and Water Emulsions. J Pet Environ Biotechnol 7: 330. doi: 10.4172/2157-7463.1000330

temperature of $70^{\circ} \mathrm{C}$. With that said, the temperature of the water bath was set at $70^{\circ} \mathrm{C}$ and a solution of $0.08 \%$ of oil-in-water was prepared and placed in the water bath to optimize the time of treatment this is presented in Table 7 (Plots 2 and 3).

From the table above, it is found that the longer the time the sample was subject to the water bath the higher (results highlighted in read) is the turbidity reduction of the sample at a constant temperature of $70^{\circ} \mathrm{C}$. That is, demulsification with water bath is directly proportional to time at constant temperature. The plot above shows the increasing turbidity reduction with respect to increasing time submerges in the water bath at constant temperature of $70^{\circ} \mathrm{C}$

\section{Centrifuge}

As with the water bath, both an optimization of the rotational

\begin{tabular}{|c|c|c|c|c|c|c|c|c|}
\hline & \multirow{2}{*}{ Time } & $\begin{array}{c}\text { Concen- } \\
\text { tration, } \\
\text { \% }\end{array}$ & RPM & \multicolumn{4}{|c|}{ Trial 1 } & \multicolumn{3}{|c|}{ Trial 2 } \\
\cline { 5 - 9 } & & Initial & Final & $\begin{array}{c}\text { \%Reduc- } \\
\text { tion }\end{array}$ & Initial & Final & $\begin{array}{c}\text { \%Reduc- } \\
\text { tion }\end{array}$ \\
\hline 5 & 0.08 & 5000 & 992 & 195 & 80 & 992 & 193 & 81 \\
\hline 8 & 0.08 & 5000 & 992 & 157 & 84 & 992 & 164 & 83 \\
\hline 10 & 0.08 & 5000 & 992 & 196 & 80 & 992 & 167 & 83 \\
\hline 15 & 0.08 & 500 & 992 & 177 & 82 & 992 & 143 & 86 \\
\hline
\end{tabular}

Note: Shaded cells highlight results with the highest turbidity reduction.

Table 8: Time optimization of the centrifugal demulsification technique.
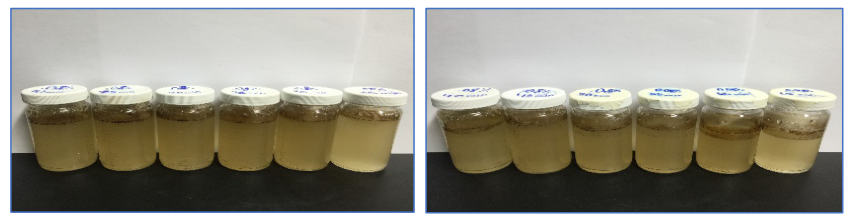

Figure 11: A $0.08 \%$ oil in water sample subjected to water bath at constant temperature at varying time (from $10 \mathrm{~min}$ to $65 \mathrm{~min}$ ).

\begin{tabular}{|c|c|c|c|c|c|c|c|c|}
\hline \multirow{3}{*}{ Time } & \multirow{3}{*}{$\begin{array}{c}\text { Concen- } \\
\text { tration, } \\
\%\end{array}$} & \multirow{3}{*}{ RPM } & \multicolumn{6}{|c|}{ Turbidity, NTU } \\
\hline & & & \multicolumn{3}{|c|}{ Trial 1} & \multicolumn{3}{|c|}{ Trial 2} \\
\hline & & & Initial & Final & $\begin{array}{l}\text { \%Reduc- } \\
\text { tion }\end{array}$ & Initial & Final & $\begin{array}{l}\text { \%Reduc- } \\
\text { tion }\end{array}$ \\
\hline 8 & 0.08 & 1000 & 942 & 456 & 52 & 942 & 395 & 58 \\
\hline 8 & 0.08 & 2000 & 942 & 352 & 63 & 942 & 314 & 67 \\
\hline 8 & 0.08 & 3000 & 942 & 230 & 76 & 942 & 210 & 78 \\
\hline 8 & 0.08 & 4000 & 942 & 144 & 85 & 942 & 183 & 81 \\
\hline 8 & 0.08 & 5000 & 942 & 210 & 78 & 942 & 197 & 79 \\
\hline 8 & 0.06 & 1000 & 671 & 274 & 59 & 671 & 290 & 57 \\
\hline 8 & 0.06 & 2000 & 671 & 147 & 78 & 671 & 184 & 73 \\
\hline 8 & 0.06 & 3000 & 671 & 161 & 76 & 671 & 112 & 83 \\
\hline 8 & 0.06 & 4000 & 671 & 153 & 77 & 671 & 99.2 & 85 \\
\hline 8 & 0.06 & 5000 & 671 & 181 & 73 & 671 & 134 & 80 \\
\hline 8 & 0.04 & 1000 & 325 & 125 & 62 & 325 & 139 & 57 \\
\hline 8 & 0.04 & 2000 & 325 & 114 & 65 & 325 & 110 & 66 \\
\hline 8 & 0.04 & 3000 & 325 & 121.1 & 63 & 325 & 68.6 & 79 \\
\hline 8 & 0.04 & 4000 & 325 & 82.9 & 74 & 325 & 78.2 & 76 \\
\hline 8 & 0.04 & 5000 & 325 & 75.7 & 77 & 325 & 57.3 & 82 \\
\hline 8 & 0.02 & 1000 & 153 & 64 & 58 & 153 & 68.9 & 55 \\
\hline 8 & 0.02 & 2000 & 153 & 39.8 & 74 & 153 & 48.9 & 67 \\
\hline 8 & 0.02 & 3000 & 153 & 23.5 & 85 & 153 & 28.3 & 82 \\
\hline 8 & 0.02 & 4000 & 153 & 19.2 & 87 & 153 & 43 & 72 \\
\hline 8 & 0.02 & 5000 & 153 & 40.2 & 74 & 153 & 37.6 & 75 \\
\hline
\end{tabular}

Note: Shaded cells highlight results with the highest turbidity reduction.

Table 9: RPM optimization of the centrifugal demulsification technique.

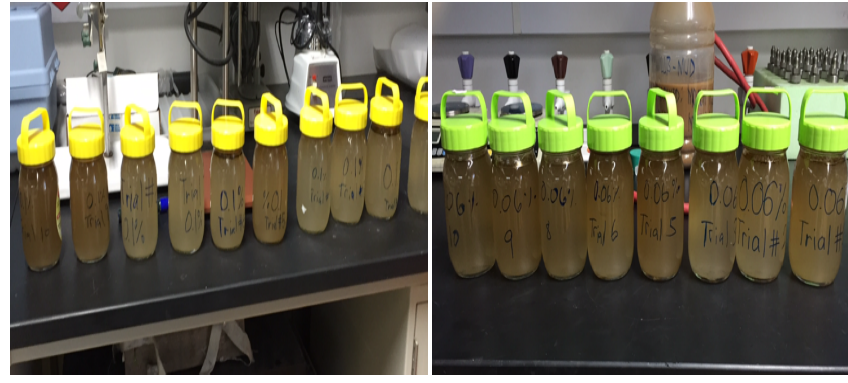

Figure 12: Oil in water emulsion samples.
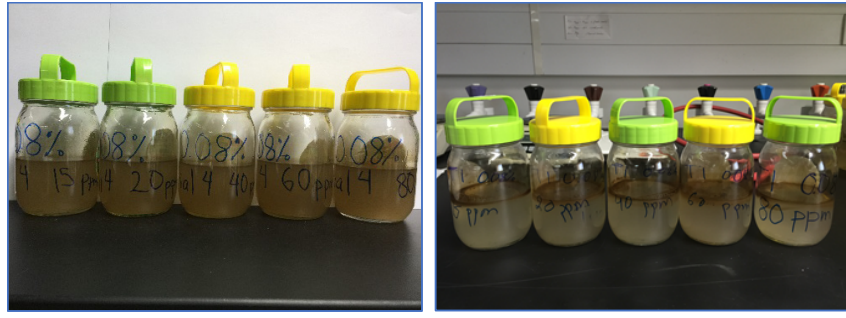

Figure 13a: A $0.08 \%$ oil in water emulsion sample before (left sample) and after (right sample) treatment.
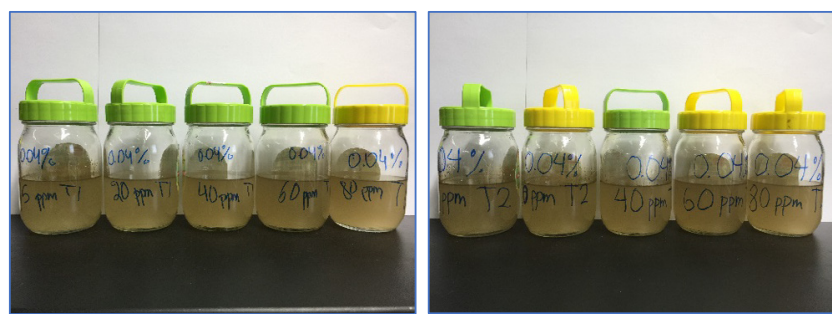

Figure 13b: A $0.04 \%$ oil in water emulsion sample before (left sample) and after (right sample) treatment.

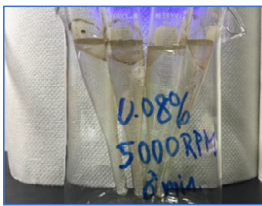

A

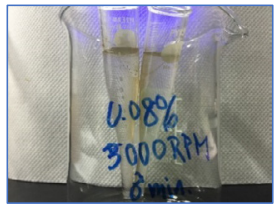

B

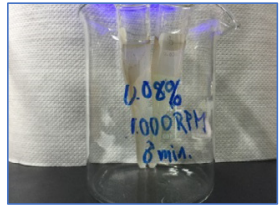

$\mathrm{C}$
Figure 14a: $A 0.08 \%$ oil in water sample subjected to the centrifuge at (A) 5000 RPM, (B) 3000 RPM and (C) 1000 RPM.

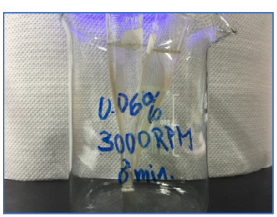

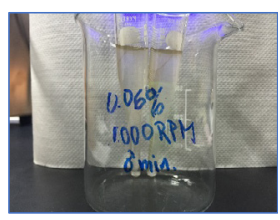

Figure 14b: A $0.06 \%$ oil in water sample subjected to the centrifuge, (A) 3000 RPM and (B) 1000 RPM.

speed and the treatment time need to be performed for this technique. To start, a solution of $0.08 \%$ oil in water was prepared and run at $5000 \mathrm{rpm}$ at different times as shown in Table 8 (Figure 11). Through that, the optimum treatment time was found to be $8 \mathrm{~min}$. From there 
Citation: Amani M, Idris M, Abdul Ghani M, Dela Rosa N, Carvero A, et al. (2017) An Experimental Study on the Application of Ultrasonic Technology for Demulsifying Crude Oil and Water Emulsions. J Pet Environ Biotechnol 7: 330. doi: 10.4172/2157-7463.1000330

an optimization of the rotational speed was done. This was through preparing solutions of different oil-in-water concentrations that were placed in the centrifuge for $8 \mathrm{~min}$ at different rpms. The results of this optimization are shown in Table 9 where it can be seen that the optimum speed is $4000 \mathrm{rpm}$ (Figures 12-18).

\section{Ultrasonication}

As the efficiency of each of the demulsification techniques has been tested, it is necessary to compare them to that of Ultrasonication. As before, both the time and amplitude of the ultrasonication treatment need to be optimized. Initially the test was run at amplitude of $100 \%$ while varying the time of treatment between $2 \mathrm{~min}$ and $14 \mathrm{~min}$ for a sample of $0.08 \%$ oil-in-water. The results of this initial run are presented in Table 10 where it can be seen that running the treatment for a time

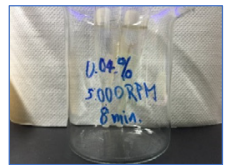

A

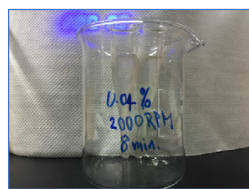

$\mathrm{D}$

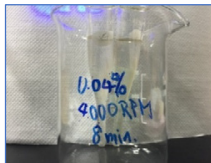

B

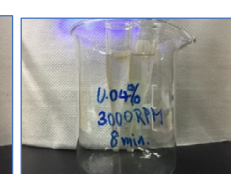

$\mathrm{C}$

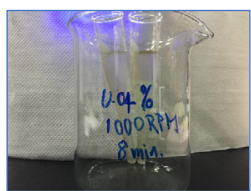

$\mathrm{E}$

Figure 14c: $A 0.04 \%$ oil in water sample subjected to the centrifuge at $(A) 5000$ RPM, (B) 4000 RPM, (C) 3000 RPM, (D) 2000 RPM and (E) 1000 RPM.

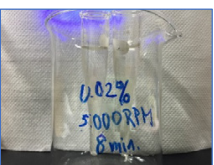

A

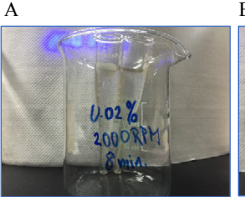

$\mathrm{D}$
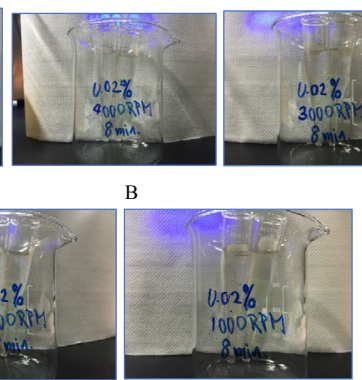

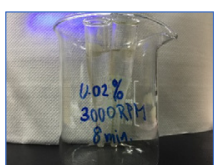

Figure 14d: $A 0.02 \%$ oil in water sample subjected to the centrifuge at (A) 5000 RPM, (B) 4000 RPM, (C) 3000 RPM, (D) 2000 RPM and (E) 1000 RPM.

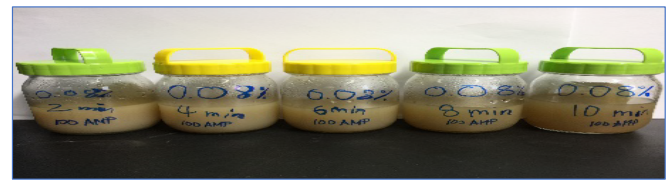

Figure 15: Time optimization of $0.08 \%$ oil in water sample by ultrasonication.

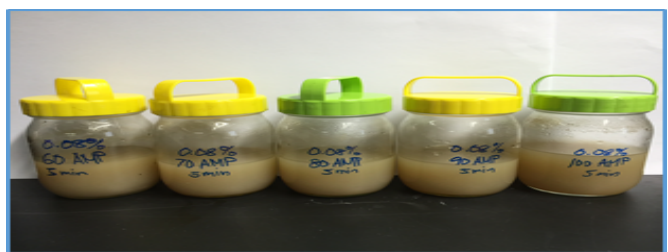

Figure 16: Amplitude optimization of $0.08 \%$ oil in water sample by ultrasonication.

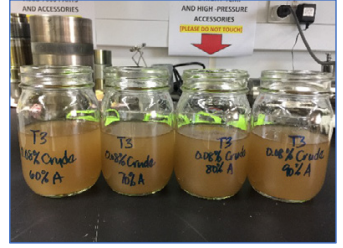

$\mathrm{A}$

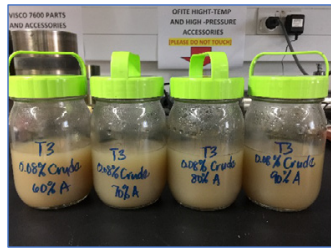

$\mathrm{B}$
Figure 17a: (A) $0.08 \%$ water in oil sample before and (B) after ultrasonication.

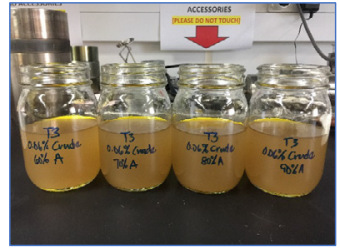

A

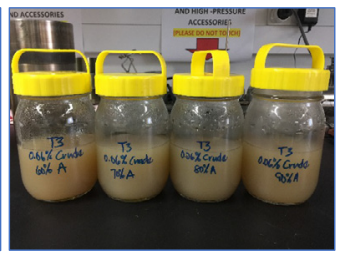

$\mathrm{B}$
Figure 17b: (A) $0.06 \%$ water in oil sample before and (B) after ultrasonication.

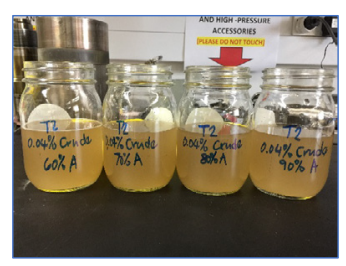

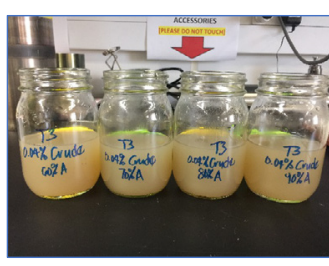

Figure 17c: (A) $0.04 \%$ water in oil sample before and (B) after ultrasonication.

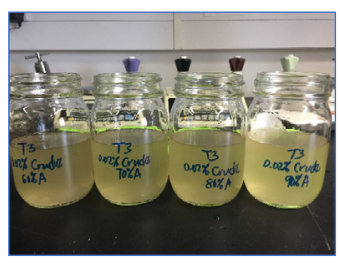

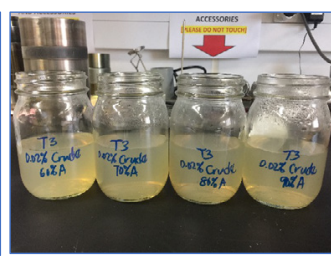

Figure 17d: (A) $0.02 \%$ water in oil sample before and (B) after ultrasonication.

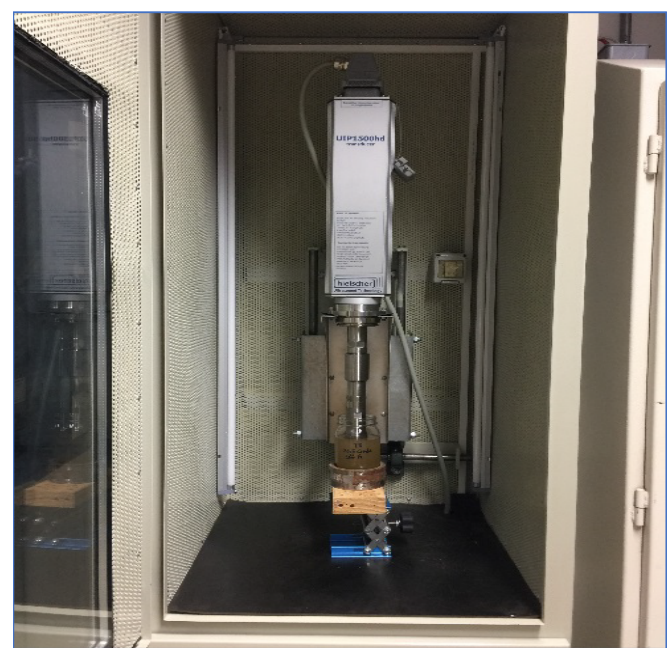

Figure 18: Hielscher ultrasonic device model UIP1500hd in sound proof cabinet. 
Citation: Amani M, Idris M, Abdul Ghani M, Dela Rosa N, Carvero A, et al. (2017) An Experimental Study on the Application of Ultrasonic Technology for Demulsifying Crude Oil and Water Emulsions. J Pet Environ Biotechnol 7: 330. doi: 10.4172/2157-7463.1000330

Page 11 of 12

\begin{tabular}{|c|c|c|c|c|c|}
\hline Time, mins & Amplitude, $\%$ & Concentration, $\%$ & Initial turbidity, NTU & Final turbidity, NTU & $\%$ Turbidity reduction \\
\hline 2 & 100 & 0.08 & 986 & OR & - \\
\hline 4 & 100 & 0.08 & 989 & 950 & 4 \\
\hline 6 & 100 & 0.08 & 961 & 787 & 18 \\
\hline 8 & 100 & 0.08 & 943 & 551 & 42 \\
\hline 10 & 100 & 0.08 & 923 & 460 & 50 \\
\hline 12 & 100 & 0.08 & 734 & 602 & 18 \\
\hline 14 & 100 & 0.08 & 699 & 587 & 16 \\
\hline
\end{tabular}

Table 10: Time optimization of ultrasonication.

\begin{tabular}{|c|c|c|c|c|c|}
\hline Time, mins & Amplitude, $\%$ & Concentration, $\%$ & Initial turbidity, NTU & Final turbidity, NTU & $\%$ Turbidity reduction \\
\hline 5 & 60 & 0.08 & 948 & OR & - \\
\hline 5 & 70 & 0.08 & 948 & 908 & 4 \\
\hline 5 & 80 & 0.08 & 948 & 790 & 17 \\
\hline 5 & 90 & 0.08 & 948 & 638 & 33 \\
\hline 5 & 100 & 0.08 & 948 & 594 & 37 \\
\hline
\end{tabular}

Table 11: Amplitude optimization of ultrasonication.

\begin{tabular}{|c|c|c|c|c|c|}
\hline \multirow{2}{*}{$\begin{array}{c}\text { Concentration } \\
\%\end{array}$} & \multirow{2}{*}{$\begin{array}{c}\text { Amplitude } \\
\text { A }\end{array}$} & \multirow{2}{*}{$\begin{array}{l}\text { Time } \\
\text { min }\end{array}$} & \multicolumn{3}{|c|}{ Turbidity, NTU } \\
\hline & & & Initial & Final & $\%$ Reduction \\
\hline \multicolumn{6}{|c|}{ Trial 1} \\
\hline 0.08 & 60 & 5 & 991 & 664 & 33 \\
\hline 0.08 & 70 & 5 & 991 & 563 & 43 \\
\hline 0.08 & 80 & 5 & 991 & 486 & 51 \\
\hline 0.08 & 90 & 5 & 991 & 575 & 42 \\
\hline 0.06 & 60 & 5 & 887 & 445 & 50 \\
\hline 0.06 & 70 & 5 & 887 & 386 & 56 \\
\hline 0.06 & 80 & 5 & 887 & 350 & 61 \\
\hline 0.06 & 90 & 5 & 887 & 375 & 58 \\
\hline 0.04 & 60 & 5 & 512 & 301 & 41 \\
\hline 0.04 & 70 & 5 & 512 & 294 & 43 \\
\hline 0.04 & 80 & 5 & 512 & 272 & 47 \\
\hline 0.04 & 90 & 5 & 512 & 261 & 49 \\
\hline 0.02 & 60 & 5 & 202 & 142 & 30 \\
\hline 0.02 & 70 & 5 & 202 & 134 & 34 \\
\hline 0.02 & 80 & 5 & 202 & 124 & 39 \\
\hline 0.02 & 90 & 5 & 202 & 125 & 38 \\
\hline \multicolumn{6}{|c|}{ Trial 2} \\
\hline 0.08 & 60 & 5 & 931 & 759 & 18 \\
\hline 0.08 & 70 & 5 & 931 & 632 & 32 \\
\hline 0.08 & 80 & 5 & 931 & 702 & 25 \\
\hline 0.08 & 90 & 5 & 931 & 833 & 11 \\
\hline 0.06 & 60 & 5 & 777 & 514 & 34 \\
\hline 0.06 & 70 & 5 & 777 & 533 & 29 \\
\hline 0.06 & 80 & 5 & 777 & 512 & 34 \\
\hline 0.06 & 90 & 5 & 777 & 532 & 32 \\
\hline 0.04 & 60 & 5 & 496 & 287 & 42 \\
\hline 0.04 & 70 & 5 & 496 & 295 & 41 \\
\hline 0.04 & 80 & 5 & 496 & 270 & 46 \\
\hline 0.04 & 90 & 5 & 496 & 272 & 45 \\
\hline 0.02 & 60 & 5 & 191 & 142 & 26 \\
\hline 0.02 & 70 & 5 & 191 & 137 & 28 \\
\hline 0.02 & 80 & 5 & 191 & 116 & 39 \\
\hline 0.02 & 90 & 5 & 191 & 119 & 38 \\
\hline \multicolumn{6}{|c|}{ Trial 3} \\
\hline 0.08 & 60 & 5 & 995 & 819 & 18 \\
\hline 0.08 & 70 & 5 & 995 & 770 & 23 \\
\hline 0.08 & 80 & 5 & 995 & 733 & 26 \\
\hline
\end{tabular}




\begin{tabular}{|c|c|c|c|c|c|}
\hline 0.08 & 90 & 5 & 995 & 732 & 26 \\
\hline 0.06 & 60 & 5 & 777 & 463 & 40 \\
\hline 0.06 & 70 & 5 & 777 & 518 & 33 \\
\hline 0.06 & 80 & 5 & 777 & 446 & 43 \\
\hline 0.06 & 90 & 5 & 777 & 473 & 39 \\
\hline 0.04 & 60 & 5 & 483 & 319 & 34 \\
\hline 0.04 & 70 & 5 & 483 & 321 & 34 \\
\hline 0.04 & 80 & 5 & 483 & 311 & 36 \\
\hline 0.04 & 90 & 5 & 483 & 301 & 38 \\
\hline 0.02 & 60 & 5 & 176 & 132 & 25 \\
\hline 0.02 & 70 & 5 & 176 & 124 & 30 \\
\hline 0.02 & 80 & 5 & 176 & 122 & 31 \\
\hline 0.02 & 90 & 5 & 176 & 123 & 30 \\
\hline
\end{tabular}

Note: Shaded cells highlight results with the highest turbidity reduction.

Table 12: Turbidity reduction with varying amplitude for different oil in water emulsion concentrations.

of 10 min causes the most reduction. Although the optimum condition was found to be $10 \mathrm{~min}$, the actual test was performed for 5 min due to the threat to safety if performed for longer times.

From the table above it was observed that the highest turbidity reduction was at the $100 \%$ amplitude. But no further test has been done for $100 \%$ amplitude due to the hazards that arise from such magnified amplitude and for safety purposes of the ultrasonic device. To proceed with, the ultrasonic test was performed for the specified time at three different amplitudes ranging from 70 to 90 . The results of the varying reduction in turbidity with amplitude are presented in Table 11. Optimum amplitude can be noted at a value of $80 \%$. With the ultrasonication demulsification technique turbidity reduction ranges between $26 \%$ to $61 \%$ which can be seen from the plots above.

\section{Conclusions}

In this project, we determined the effectiveness of the thermal (water bath), mechanical (centrifuge), chemical (demulsifiers), and ultrasonication as means of demulsification by applying it on various oil-in-water mixtures, and measuring the turbidities of each before and after application.

1. Based on our attempt to establish base turbidity values for each oil-in-water mixture, it was found that the turbidity of these mixtures was difficult to keep sufficiently constant. This is because the turbidity is affected by some uncontrollable factors. These factors include the imprecision of our liquid transferring equipment and instrument to measure turbidity.

2. In finding an optimum emulsion breaker concentration, consistent trend cannot be identified. However, out of the three oil-inwater mixtures tested, the optimum emulsion breaker concentration decreased as percent oil in water decreased. In other words, the lower the oil to water ratio, the lower the amount of demulsifier should be added.

3. The most reduction in turbidity for water bath was attained at the highest temperature $\left(70^{\circ} \mathrm{C}\right)$ and longest duration $(70 \mathrm{~min})$. Hence, the higher the temperature and longer the duration for thermal demulsification, the more effective.

4. The optimum time for centrifuge method was found to be $8 \mathrm{~min}$. Hence the optimum duration for such method is not always the longest. The optimum centrifuge speed was found to be $4000 \mathrm{rpm}$. Again, this is not the highest rotational speed. So, the optimum time and rotational speed must be determined for specific oil and water emulsions.

5. Optimum time for ultrasonication is $10 \mathrm{~min}$, which again isn't the longest duration (14 min). But max reduction in turbidity is achieved at maximum amplitude, in this case at $100 \%$.
6. As data shows, there was a larger turbidity reduction on the centrifuge technique than any other technique.

7. Ultrasonication does reduce the turbidity of the sample ranging from $20 \%$ up to $60 \%$.

8. $100 \%$ amplitude was not done in the study for the reason that the machine was having a history of being damaged at $100 \%$ amplitude.

9. Turbidity reading variation was observed on different samples of the same oil-in-water solution concentration.

\section{Nomenclature}

T: Period, seconds,

f: Frequency, Hertz

F: Force, Newton

k: Proportionality constant for Hooke's law, Newton/meters

x: Displacement, meters

$\omega$ : Amplitude, $1 /$ seconds

$\varphi$ : Phase angle, dimensionless

t: Time, seconds

$\pi$ : Irrational constant pi

v: Wave speed, $\mathrm{m} / \mathrm{s}$

$\mu$ : Inertial quantity, unit varies

\section{Acknowledgment}

"This report was made possible by a UREP award [UREP No: 18-102-2-042] from the Qatar National Research Fund (a member of The Qatar Foundation). The statements made herein are solely the responsibility of the authors".

\section{References}

1. Singh B, Padney B (1992) Ultrasonication for breaking water-in-oil emulsions Proc Indian natn Sci Acad 58: 181-194.

2. Schoeppel RJ, Howard AW (1966) Effect of ultrasonic irradiation on coalescense and separation of crude oil-water emulsions. Society of Petroleum Engineers SPE-1507-MS.

3. Gholam R (2014) Two-stage ultrasonic irradiation for dehydration and desalting of crude oil: A novel method. Chemical Engineering and Processing.

4. Kokal S (2008) Crude oil emulsions: Everything you wanted to know but were afraid to ask. Society of Petroleum Engineers.

5. Islam MR, Genyk R, Malik Q (2000) Experimental and mathematical modelling of ultrasonic treatments for breaking oil-water emulsions. Petroleum Society of Canada. 